\title{
A MORDELL-WEIL GROUP OF RANK 8, AND A SUBGROUP OF FINITE INDEX
}

\author{
CHARLES F. SCHWARTZ
}

It is well known [c.f. Kas] that every elliptic surface, with geometric genus 0 , is given by a Weierstrass equation of the form

$$
y^{2}=4 x^{3}-\sum_{i=0}^{4} a_{i} u^{i} x-\sum_{j=0}^{6} b_{j} u^{j}
$$

(relative to a suitable parameter, $u$, for the base) where the $a$ 's and $b$ 's are constants. For sufficiently general choices of $a$ 's and $b$ 's, the MordellWeil group (i.e., the group of solutions $(x, y)$, with $x$ and $y$ rational functions of $u$ ) has rank 8 .

We will find, for a specific equation of this form,

$$
y^{2}=4\left(x^{3}-u^{4} x+1\right)
$$

8 solutions that generate a subgroup of index 4 in the Mordell-Weil group of the fibration given by this equation. We do this using the Cox-Zucker Machine. We then use this result to draw certain conclusions concerning the general case, and to make certain conjectures.

\section{§1. The algorithm of Cox and Zucker (AKA, The Cox-Zucker Machine)}

The purpose of this section is to provide, not a complete description of the algorithm of Cox and Zucker, but rather, a brief summary of their technique. See [1] for details.

Let $E \stackrel{f}{\longrightarrow} B$ be an elliptic fibration with geometric genus zero; let $\sigma_{0}: B \rightarrow E$ be the zero section, i.e., the identity element of the MordellWeil group; let $($,$) be the intersection product on the space of 1-cycles$ on the surface, $E$; and let $S \subset B$ be the finite set of points of $B$, above which lie the singular fibers.

Received June 21, 1982. 
Define a symmetric bilinear form on the Mordell-Weil group of $E$, by

$$
\left\langle\sigma_{i}, \sigma_{j}\right\rangle=-\left(\sigma_{i}-\sigma_{0}, \sigma_{j}-\sigma_{0}\right)-\sum_{s \in S} \text { L.C.F. }
$$

(where L.C.F. stands for local correction factors, rational numbers which are read from [1], table 1.19, and which depend on the components of the fiber above $s$, of the Néron model, through which the sections pass).

Let $m_{s}$ be the number of components of multiplicity one in the fiber, above $s$, of the Néron model; let $N$ be the number of elements of the torsion subgroup of the Mordell-Weil group; and let $r$ be the rank of the Mordell-Weil group.

Theorem (Cox and Zucker, [1]): $\left\{\sigma_{1}, \cdots, \sigma_{r}\right\}$ is a basis of the MordellWeil group if and only if the determinant

equals

$$
\operatorname{det}\left(\left\langle\sigma_{i}, \sigma_{j}\right\rangle\right)
$$

$$
N^{2} / \prod_{s \in S} m_{s}
$$

CoRollary. $\quad\left\{\sigma_{1}, \cdots, \sigma_{r}\right\}$ generates a subgroup of index $k$ if and only if

$$
\operatorname{det}\left(\left\langle\sigma_{i}, \sigma_{j}\right\rangle\right)=N^{2} k^{2} / \prod_{s \in S} m_{s} \text {. }
$$

§ 2. The example $y^{2}=4\left(x^{3}-u^{4} x+1\right)$

Note that the elliptic surface given by

$$
y^{2}=4\left(x^{3}-u^{4} x+1\right)
$$

may be obtained by a base extension, $v=u^{2}$, from either of the surfaces

$$
y^{2}=4\left(x^{3}-v^{2} x+1\right) \quad \text { or } \quad y^{2}=4\left(x^{3}-x+v^{3}\right) ;
$$

and each of these is of the form

$$
y^{2}=4 x^{3}-Q(v) x-C(v),
$$

where $Q(v)$ and $C(v)$ are, respectively, quadratic and cubic polynomials in $v$. We now describe a basis of the Mordell-Weil group of an elliptic surface given by an equation of the form (3); we then show, if you have two different surfaces of this type, which become equivalent under this type of base extension, then the bases of the Mordell-Weil groups of these surfaces lift to a basis of a subgroup of index 4 of the Mordell-Weil group of the new surface. 
Let $E \stackrel{f}{\longrightarrow} B$ be the elliptic surface given by

$$
\begin{aligned}
y^{2} & =4 x^{3}-Q(u) x-C(u) \\
& =4 x^{3}-\left(q_{2} u^{2}+q_{1} u+q_{0}\right) x-\left(c_{3} u^{3}+c_{2} u^{2}+c_{1} u+c_{0}\right) .
\end{aligned}
$$

Then we can find sections of the fibration by setting $x=A \mathrm{u}+B$, and finding $A$ and $B$ so that the resulting cubic,

$$
4(A u+B)^{3}-Q(u)(A u+B)-C(u)
$$

is a perfect square. In particular,

$$
0=4 A^{3}-q_{2} A-c_{3}
$$

and

$$
\begin{aligned}
0= & \left(12 A B^{2}-q_{1} B-q_{0} A-c_{1}\right)^{2} \\
& -4\left(12 A^{2} B-q_{2} B-q_{1} A-c_{2}\right)\left(4 B^{3}-q_{0} B-c_{0}\right) .
\end{aligned}
$$

In general, the first equation yields 3 distinct solutions for $A$; and for each of these, the second equation yields 4 solutions for $B$. Let $A$ and $A^{\prime}$ be distinct solutions of equation (4); and let $B_{1}, B_{2}, B_{3}$, and $B^{\prime}$ be numbers so that $\left(A, B_{1}\right),\left(A, B_{2}\right),\left(A, B_{3}\right)$ and $\left(A^{\prime}, B^{\prime}\right)$ are distinct solutions of the pair of equations, (4) and (5). Let $\sigma_{i}=\left(x_{i}, y_{i}\right)$ be a section with $x_{i}=A u+B_{i}$, for $i=1,2$, or 3 ; and let $\sigma_{4}=\left(x_{4}, y_{4}\right)$ be a section with $x_{4}=A^{\prime} u+B^{\prime}$.

Lemma 2.1. Suppose the elliptic surface $E \stackrel{f}{\longrightarrow} B$ is given by an equation

$$
y^{2}=4 x^{3}-Q(u) x-C(u)
$$

and suppose that the J-invariant is non-constant, and that the singular fibers of the Néron model consist of:

6 fibers of type $I_{1}$, above finite values of $u$; and

1 fibers of type $I_{0}^{*}$, above $u=\infty$ Then $\left\{\sigma_{i} \mid i=1,2,3,4\right\}$ is a basis of the Mordell-Weil group of this surface.

Proof. Using a formula of Shioda [3], one can show that the MordellWeil rank is 4.

It is easy to compute that $\prod m_{s}=4$.

We prove that the torsion subgroup consists of $\left\{\sigma_{0}\right\}$ only, by considering the bilinear form applied to $\tau$ and itself, with $\tau$ torsion and $\neq \sigma_{0}$. On the one hand $\langle\tau, \tau\rangle=0$; but on the other hand, $\tau$ must pass through 
some non-identity component of a singular fiber of the Néron model, and the only non-identity components are those of fiber of type $I_{0}^{*}$, above $u=\infty$. The local correction factor, from table (1.19) of [1], is 1 . So

$$
\langle\tau, \tau\rangle=-\left(\tau-\sigma_{0}, \tau-\sigma_{0}\right)-1=-\left(-1-2 \tau \cdot \sigma_{0}-1\right)-1,
$$

which cannot be zero. (See [1], §2, for details.) So

$$
N^{2} / \prod m_{s}=1 / 4 \text {. }
$$

One next computes the matrix

$$
\begin{aligned}
& \left(\left\langle\sigma_{i}, \sigma_{j}\right\rangle ; 1 \leq i, j \leq 4\right) \text { to be } \\
& \left.\qquad \begin{array}{rrrr}
1 & 0 & 0 & \pm \frac{1}{2} \\
0 & 1 & 0 & \pm \frac{1}{2} \\
0 & 0 & 1 & \pm \frac{1}{2} \\
\pm \frac{1}{2} & \pm \frac{1}{2} & \pm \frac{1}{2} & 1
\end{array}\right)
\end{aligned}
$$

The sign of $\left\langle\sigma_{i}, \sigma_{4}\right\rangle$ is determined by whether the sections $\sigma_{i}$ and $\sigma_{4}$ intersect; but since the matrix is symmetric, these signs turn out to be irrelevant, and the determinant of the matrix is $1 / 4$.

Q.E.D.

(For the sufficiency of the hypothesis to imply the existence of the sections $\sigma_{i}$, see [2]. At any rate, it is clear that for almost all elliptic surfaces given by equations of the form (3), the singular fibers of the Néron model are as in the hypothesis of the lemma, and that the 12 sections described will exist and be distinct.)

Notice that if

$$
y^{2}=4 x^{3}-G_{2}(u) x-G_{3}(u)
$$

is of the form (3), then

$$
y^{2}=4 x^{3}-u^{2} G_{2}(u) x-u^{3} G_{3}(u)
$$

can also be put in the form (3).

Suppose that $E_{1} \stackrel{f_{1}}{\longrightarrow} B_{1}$ and $E_{2} \stackrel{f_{2}}{\longrightarrow} B_{2}$ are the elliptic surfaces given by

$$
y^{2}=4 x^{3}-Q(v) x-C(v), \text { and } y^{2}=4 x^{3}-v^{2} Q(v) x-v^{3} C(v) ;
$$

and suppose that we have bases $\left\{\sigma_{1}, \sigma_{2}, \sigma_{3}, \sigma_{4}\right\}$ and $\left\{\sigma_{1}^{\prime}, \sigma_{2}^{\prime}, \sigma_{3}^{\prime}, \sigma_{4}^{\prime}\right\}$ for the Mordell-Weil groups of $E_{1}$ and $E_{2}$, respectively, of the type described in the lemma. Let $E$ be the elliptic surface obtained from either $E_{1}$ or $E_{2}$ by the base extension, $v=u^{2}$. 
Theorem 2.2. For almost all pairs of elliptic surfaces, $E_{1}$ and $E_{2}$, as above, the bases of the Mordell-Weil groups of $E_{1}$ and $E_{2}$ lift to a basis of a subgroup (of index 4) of the Mordell-Weil group of $E$.

Proof. One can use Shioda [3] to show that the Mordell-Weil rank of the surface $E$ is 8 .

The torsion subgroup of the Mordell-Weil group of $E$ is seen to be $\left\{\sigma_{0}\right\}$ only, since there are no non-identity components of singular fibers of the Néron model; and each $m_{s}=1$, since all the singular fibers are of type $I_{1}$; so

$$
N^{2} / \prod m_{s}=1
$$

We now find it necessary to pay a bit of attention to the details of the form of the sections.

If $\sigma_{i}=\left(x_{i}, y_{i}\right)$ is a section of the surface $E_{1}$ of the type described in the lemma, then $x_{i}$ and $y_{i}$ are both linear polynomials in $v$. Upon the base extension $v=u^{2}$ they both become quadratic polynomials in $u$, and in addition, they are both even functions.

If $\sigma_{i}=\left(x_{i}^{\prime}, y_{i}^{\prime}\right)$ is a section of the surface $E_{2}$ of the type described in the lemma, then $x_{i}^{\prime}$ and $y_{i}^{\prime}$ are, respectively, a quadratic (without a constant term) and a cubic (without linear or constant term). Upon the base extension $v=u^{2}, x_{i}^{\prime}$ and $y_{i}^{\prime}$ become even functions of $u$, of degree 4 and 6 , respectively, which satisfy the equation $y^{2}=4 x^{3}-u^{4} Q\left(u^{2}\right) x-u^{6} C\left(u^{2}\right)$; but we can reduce this equation by dividing the coefficient of $x$ and the "constant" term by $u^{4}$ and $u^{6}$, respectively, and dividing $x_{i}^{\prime}$ and $y_{i}^{\prime}$ by $u^{2}$ and $u^{3}$ respectively. Then $x_{i}^{\prime}$ is a quadratic polynomial (in $u$ ) without linear term, and $y_{i}^{\prime}$ is a cubic polynomials (in $u$ ) without quadratic or constant term. In particular $x_{i}^{\prime}$ is an even function and $y_{i}^{\prime}$ is an odd function of $u$.

Using the parity of the functions, $x_{i}, y_{i}, x_{i}^{\prime}$, and $y_{i}^{\prime}$, we find that the matrix of the bilinear form looks like

$$
\left(\begin{array}{rrrrrrrr}
2 & 0 & 0 & \pm 1 & & & & \\
0 & 2 & 0 & \pm 1 & & & & \\
0 & 0 & 2 & \pm 1 & & & & \\
\pm 1 & \pm 1 & \pm 1 & 2 & & & & \\
& & & & 2 & 0 & 0 & \pm 1 \\
& & & & 0 & 2 & 0 & \pm 1 \\
& & & & 0 & 0 & 2 & \pm 1 \\
& & & & \pm 1 & \pm 1 & \pm 1 & 2
\end{array}\right)
$$


which has determinant 16 . Hence the subgroup generated by these sections has index 4 in the Mordell-Weil group.

Q.E.D.

The equivocation "almost all" in the statement of the theorem arises from the following: Let $\sigma_{i}=\left(x_{i}, y_{i}\right)$, and $\sigma_{j}=\left(x_{j}^{\prime}, y_{j}^{\prime}\right)$, with $x_{i}, y_{i}$, and $x_{j}^{\prime}$ even quadratic functions, and $y_{j}^{\prime}$ an odd cubic function; then the equation $x_{i}=x_{j}$, typically has 2 distinct solutions $u= \pm u_{0}$; then exactly one of the statements

$$
\left\{y_{i}\left(u_{0}\right)=y_{j}^{\prime}\left(u_{0}\right), y_{i}\left(-u_{0}\right)=y_{j}^{\prime}\left(-u_{0}\right)\right\}
$$

will hold because of the parity of the functions, except in cases where $y_{i}\left(u_{0}\right)=y_{j}^{\prime}\left(u_{0}\right)=y_{i}\left(-u_{0}\right)=y_{j}^{\prime}\left(-u_{0}\right)=0$. These cases can arise, but I have evidence to suggest that, in these cases, the singular fibers of the Neron models of $E_{1}$ and $E_{2}$ are not as hypothesized in Lemma 2.1.

Now consider the elliptic surface given by

$$
y^{2}=4\left(x^{3}-u^{4} x+1\right) .
$$

It can easily be checked that this surface satisfies the hypothesis of Theorem 2.2. Then we get the following:

Corollary 2.3. Let $c$ be a real root of

$$
3 c^{4}-6 c^{2}-1=0,
$$

and let $c$ be an imaginary root. Then these 8 sections generate a subgroup of the Mordell-Weil group, of index 4:

$$
\begin{aligned}
& \{(0,2), \\
& \left(-1,2 u^{2}\right), \\
& \left(e^{\pi i / 3}, 2 e^{2 \pi i / 3} u^{2}\right), \\
& \left(u^{2}, 2\right), \\
& \left(-1+c u^{2}, \sqrt{c}\left(\left(3-3 c^{2}\right) u+\left(3 c^{3}-5 c\right) u^{3}\right)\right), \\
& \left(-1-c u^{2}, \sqrt{-c}\left(\left(3-3 c^{2}\right) u+\left(5 c-3 c^{3}\right) u^{3}\right)\right), \\
& \left(-1+c^{\prime} u^{2}, \sqrt{c^{\prime}}\left(\left(3-3 c^{\prime 2}\right) u+\left(3 c^{3}-5 c^{\prime}\right) u^{3}\right)\right), \\
& \left.\left(-e^{2 \pi i / 3}+c u^{2}, \sqrt{c}\left(\left(3-3 c^{2}\right) e^{2 \pi i / 3} u+\left(3 c^{3}-5 c\right) u^{3}\right)\right)\right\}
\end{aligned}
$$

\section{§ 3. The general case}

To treat the general case,

$$
y^{2}=4 x^{3}-\sum_{i=0}^{4} a_{i} u^{i} x-\sum_{j=0}^{6} b_{j} u^{j}
$$


we need the following lemma.

Lemma 3.1. The sixth degree polynomial

$$
\alpha u^{6}+\beta u^{5}+\gamma u^{4}+\delta u^{3}+\varepsilon u^{2}+\zeta u+\eta, \quad \alpha \neq 0
$$

is a perfect square, in $C(u)$, if and only if these three polynomial equations in the coefficients are satisfied:

$$
\begin{aligned}
& 64 \alpha^{3} \varepsilon=4 \beta\left(8 \alpha^{2} \delta-\beta\left(4 \alpha \gamma-\beta^{2}\right)\right)+\left(4 \alpha \gamma-\beta^{2}\right)^{2} \\
& 64 \alpha^{4} \zeta=\left(4 \alpha \gamma-\beta^{2}\right)\left(8 \alpha^{2} \delta-\beta\left(4 \alpha \gamma-\beta^{2}\right)\right) \\
& 256 \alpha^{5} \eta=\left(8 \alpha^{2} \delta-\beta\left(4 \alpha \gamma-\beta^{2}\right)\right)^{2} .
\end{aligned}
$$

We now find sections of the elliptic surface given by (1) (or, at least, we write the polynomial equations satisfied by the coefficients of certain sections) as follows:

Suppose $x=A u^{2}+B u+C$, i.e., the degree of $x$ is less than 3. Then

$$
4 x^{3}-\sum_{i=0}^{4} a_{i} u^{i} x-\sum_{j=0}^{6} b_{j} u^{j}
$$

is a sixth degree polynomial. Applying the lemma, this is a square if and only if the three equations (in $A, B$ and $C$, with coefficients that are polynomials in the $a$ 's and $b$ 's) are satisfied.

Notice that the solutions in Section 2 all had $\operatorname{deg} x \leq 2$. Then I claim the following:

THEOREM 3.2. For almost all choices of a's and b's, the solutions with $\operatorname{deg} x \leq 2$ generate a subgroup of the Mordell-Weil group (of (1)) of finite index.

Proof. If you view $A, B$ and $C$ as being multivalued functions of the $a$ 's and $b$ 's (no longer just constants), then the 8 solutions in Section 2 are the specializations of solutions in the general case. The solutions (in the case of variable $a$ 's and $b$ 's) must span a subgroup of rank 8 since they span a subgroup of rank 8 upon specialization to the case in Section 2. But then they span a subgroup of rank 8 , and hence generate a subgroup of finite index, upon specialization, for almost all choices of $a$ 's and $b$ 's.

CONJECTURE. For all choices of a's and b's (such that the J-invariant of the elliptic surface is non-constant), the solutions with deg $x \leq 2$ and $\operatorname{deg} y \leq 3$ span a subgroup of the Mordell-Weil group of finite index. 
As nearly as we can tell, this has to be checked case by case. The problem that may arise is that the rank spanned by the solutions of the required type may drop, but the Mordell-Weil rank not drop, for some specialization.

Note added in Proof. Theorem 3.2 may be strengthened to state that the sections with deg $x \leq 2$ generate the entire Mordell-Weil group, for almost all choices of $a^{\prime}$ s and $b^{\prime}$ s.

Proof. In [4], we give an example with a basis of this type.

\section{REFERENCES}

[1] Cox, D. A. and Zucker, S., Intersection numbers of sections of elliptic surfaces, Invent. Math., 53 (1979), 1-44.

[2] Schwartz, C. F., On a family of elliptic surfaces with Mordell-Weil rank 4, to appear.

[ 3 ] Shioda, T., On elliptic modular surfaces, J. Math. Soc. Japan, 24 (1972), 20-59.

[4] Schwartz, C. F., An elliptic surface of Mordell-Weil rank 8 over the rational numbers, to appear.

Department of Mathematics

Rider College

P.O. Box 6400, Lawrenceville

New Jersey 08640

USA 